\title{
TTILE: CHARACTERIZATION OF A DEFECTIVE INTERFERING RNA THAT CONTAINS A MOSAIC OF A PLANT VIRAL GENOME
}

\author{
PIs: TJ. Morris and A.O. Jacisson \\ Departinent of Plant Pathology, \\ University of California, \\ Berkeley, California, 94720.
}

\author{
DOE/ER/13908--TI \\ DE92 015221
}

\section{INTRODUCTION}

Our lab was the first to describe and characterize a deiective interfering RNA (DI RNAs or DIs) in association with a small RNA plant virus (Hillman et al., 1987). The features of the DIs that we discovered in infections of tomato bushy stunt virus were compatible with the properties of DIs identified in many animal virus infections. Animal virologists have generally recognized the importance of studying DIs because they are invaluable tools for identifying cis-acting sequences important in virus multiplication and because they offer the opportunity to elucidate mechanisms involved in viral persistence and disease attenuation. Hence our discovery offered a comparably valuable tool for use in plant virus studies for the first time. Since then, we have also discovered the second example of plant viral DI RNAs associated with turnip crinkle virus (TCV), a virus structurally related to TBSV (Li et al., 1989). The discovery of DIs in plant viruses formed the basis the DOE grant which was awarded in 1988. We proposed a thorough characterization of this unique class of symptom modulating RNAs with the cverall objective of identifying viral RNA nucleotide. sequences involved in such fundamental processes as virus replication and encapsidation as well as the degree of symptom expression resulting from the viral-DI-host interaction. The proposed research focused on the molecular characterization of the DI RNAs and the helper virus. We had demonstrated that the DIs were collinear deletion mutants of the genome of a cherry strain of tomato bushy stunt virus (TBSV). We had also shown that these low molecular weight RNAs interfered with the helper plant virus and modulated disease expression by preventing th: development of a lethal necrotic disease in susceptible host plants. We also suggested that by exploring the mechanisms associated with the symptom attenuation effect, we might be able to devise novel strategies useful for engineering viral disease resistance. Recent results establishing TBSV as the likely etiologic agent of tomato plant decline lends urgency to such application in view of the serious nature of this disease froblem in the irrigated desert areas of California (Gerik \& Duffus, 1989).

To accomplish this, we defined 5 major objectives for the grant period. We have completed three of the proposed research objectives and have made substantial progess on the other two. A reiteration of each of the proposed objectives follows along with a brief statement of the specific accomplishments completed or expected to be completed before the end of the grant period. Additional details of the research are provided in the attached reprints and preprints.

OBJECTTVE 1) To complete the determination of the genomic sequence of TBSV as well as the nucleotide sequence of several DI RNA molecules that differ in size and degree of disease interference.

The Helper Virus Sequence: This objective has been completed. An essential priority was the completion of the cloning and sequencing of the genome of the helper virus because detailed knowledge of the helper virus was an essential prerequisite to understanding the origin and evolution 
of the DI RNAs. We made CDNA clones of the entire genome of the cherry strain of tomato bushy stunt virus (TBSV-cherry), completed the entire genomic sequence and defined gene expression by mapping the sequence origins of the two 3' co-terminal subgenomic RNAs. A paper describing much of the cloning and the 3'-proximal genome organization of the virus was published (Hillman et al., 1989) and a second paper reporting the complete sequence of the viral genome and a comparison of TBSV to other related small RNA viruses is in press (Hearne et al., 1990). The sequence data shows that two nested open reading frames (ORFs) occur in the most 3' proximal location on the genome suggesting that the $0.9 \mathrm{~kb}$ subgenomic RNA potentially encodes two polypeptides of 19,397 and 21,610 daltons. Comparison of the amino acid sequence of a potential translation product of 41,024 daltons encoded by the first open reading frame (ORF) of the $2.2 \mathrm{~kb}$ subgenomic RNA with the published capsid protein amino acid sequence of the BS.3 strain of TBSV indicates that the 2.2 $k b$ subgenomic RNA encodes the capsid protein. The TBSV coat protein cistron is located internaily on the genome and thus its genetic organization differs from that reported for most other small, spherical viruses with monopartite genomes (see Fig.2 of proposal).

DI RNA Sequences: In addition to the ncnsensus sequence determination of the DI RNAs originally characterized, we have now complet \& the sequence analysis of 18 different DI clones from 4 separate DI populations. These DIs represent novel species generated de novo from the helper virus genome and their sequence determination has provided important clues atout what the essential sequences are for DI function. We have also sequenced DIs in the process of evolving upon continued host passage. These sequence results have provided a comprehensive picture of the molecular structure of the TBSV DIs and important clues as to their origin (Knorr et al., in preparation).

OBJECTTVE 2) To construct recombinant, full-length cDNA clones of DI RNAs and the TBSV genomic FNA in the desired orientation precisely inserted at the transcription initiation site of the T/ promotor. These clones would be used to produce infectious RNA transcripts in vitro using T7 RNA polymerase.

Infectious Transcripts of TBSV: We have recently completed the construction of clones of the entire TBSV genome from which infectious transcripts can be derived (Hearne et al., 1990). This is an important breakthrough in our proposed DI research because it has provided an essential reagent ior subsequent DI studies; a cloned and completely characterized helper virus genome. This will now allow us to precisely control the purity of the inoculum and to define specific mutants that will permit us to trace the origin of DI sequences. Specific details are provided in the attached reprint and preprint and are not reiterated here.

Infectious Clones of the DI RNAs: The cloning and sequencing of the DI RNAs proved more difficult than predicted and the original approaches proposed in the grant were eventually abandoned. Instead, we devised a PCR based strategy designed to isolate full-length CDNA of the Dis behind a $T 7$ promotor sequence such that RNA transcripts could be produced from each PCR amplified cDNA. This approach greatly facilitated the cloning of JI RNAs from both virion RNA and cellular RNA preparations and it permitted us to test each clone for biological activity prior to sequencing. The protocol is diagrammed in Figure 3 of the grant proposal. First strand cDNA synthesis was initiated on the template RNA using the $3^{\prime}$ proximal transcription primer indicated and this CDNA was then amplified by PCR using a 3' cloning primer to add restriction sites at the 3'end to facilitate cloning and a 5 'terminal primer containing an Apa I site for cloning and a $\$ 7$ promotor sequence to permit RNA transcription from the 5'proximal end of the viral DI cDNA clone. These results, along with the ability to produce. DI-free inocula from clones of the complete viral genome, marks a most significant achievement in the development of the TBSV-DI experimental system. It 
means we can now, for first time, use complctely sequenced and defined biological reagents in all of our future studies.

OBJECTIVE 3) To directly address the origin of DI RNAs from the helper virus genome and to evaluate the evolution of DI RNAs upon continued host passage.

Substantial progress was made during the previous grant period toward understanding the origin and evolution of the DI RNAs. Experiments performed prior to the availability of infectious transcripts of either the DI or the helper virus genome provided very convincing evidence that the TBSV associated DIs could be generated de novo from the viral genome.

An important aspect of Objectives 1 and 2 involved a more complete characterization of DI RNAs associated with our TBSV isolate. At an early stage in these studies, we noted the appearance of novel DI species in a DI-free isolate of the virus. The experimental approach used to address the origin of animal virus DIs has generally involved passage of clonal virus inocula at high multiplicity (m.o.i.). Size and sequence heterogenaity in the DI populations resulting from such experiments has been used as evidence in support of de novo generation of DIs from the helper virus genome rather than the selection of pre-existing DI molecules. We performed an analogous experiment to demonstrate the origin of novel TBSV DI RNAs as an important component of investigating the biological variability of the DI RNAs and generated novel DI RNAs de novo by serial passage of TBSV at high multiplicity in two different host types. A DI-free preparation of TBSV RNA was inoculated to the local lesion host Nicoi ina glutinosa and 12 single lesion isolates were selected and passed on the systemic hosts, $N$ clevelandii and $N$. benthamiana. Six of the isolates were maintained in each host by weekly serial passages without dilution (high m.o.i.) and two were passed at a sap dilution of $1 / 200$ (low m.c.i.). Symptom attenuation was evident by passage 11 in all of the high m.o.i.

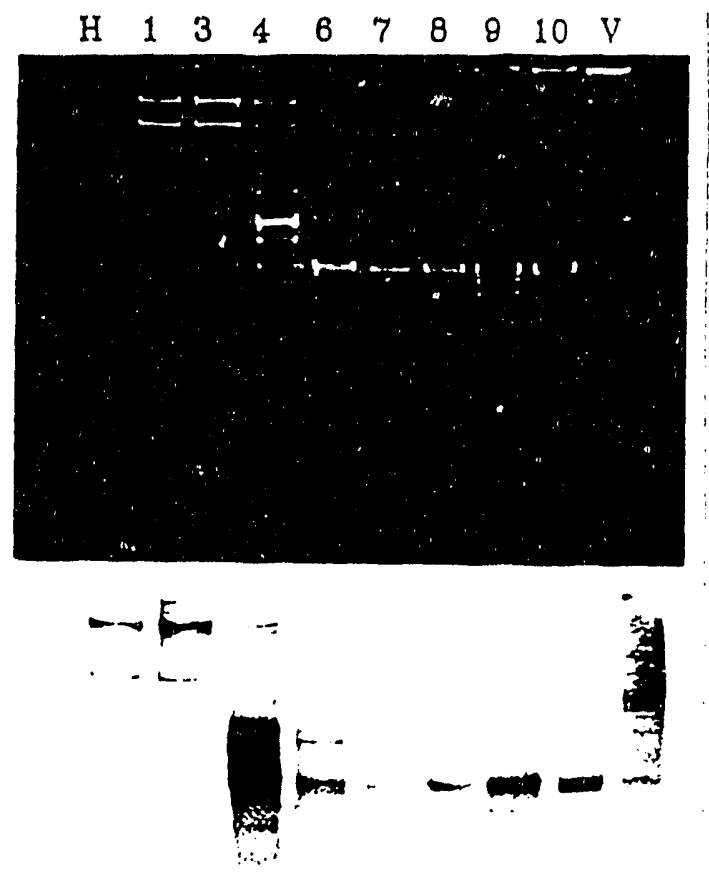
inoculations while each of the 4 low m.o.i. passage isolates displayed the same level of lethal necrosis associated with the parent isolate. Virus was purified at passage \#11 from each of the 12 high m.o.i. isolates and viral RNA was isolated and analyzed for the presence of encapsidated DI RNAs by electrophoresis in 5\% acrylamide/7M urea gels under denaturing conditions. Total cellular KNAs were also isolated from infected tissue and disease specific RNAs were analyzed after fractionation with $3 \mathrm{M} \mathrm{LiCl}$ by electrophoresis in nondenaturing 5\% acrylamide gels. Unique DI species were associated with each of the high m.o.i. passaged isolates.

The appearance and evolution of the DI RNAs was also monitored by PAGE analysis of RNAs isclated at 7 days p.i. after each passage. Northern blot hybridizations of $3 \mathrm{M}$ lithium chloride soluble RNA fractions isolated from an $N$. benthamiana (\#B-10) isolate after serial high m.o.i. passage is illustrated in Fig. 1 above. Ianes 1 through 10 contain RNA isolated from plants at the 
respective passages, lane $H$ conlains RNA from uninoculated plants and lane $V$ contains RNA isolated from virions. The RNAs were separated on a $5 \%$ acrylamide gel, transferred to nylon membrane and hybridized to a nick translated clone of the $3^{\prime}-$ end of the viral genome. In all cases, the appearance of symptom atlenuation was coincident with the appearance of DI RNA molecules in the plants determined by Northern blot analysis. Note the marked sequence heterogeneity and size evolution in successive passages in Fig.1.

Each of the 12 single lesion TBSV isolates contained symptom attenuating, low-molecular weight RNA species after high m.o.i. passage. Each hybridized to probes containing both 5' and $3^{\prime}$ genomic RNA sequences and was judged to be a defective interlering RNA that had been generated de novo from the parent viral genome. Each possessed a distinctively different electrophoretic mobility in denaturing gels and different degrees of symptom altenuation were also evident for different isolates. The likelihood that there was a preferred sequence motif selected in the high multiplicity passage experiment is suggested from the fact that all of generated DI RNAs were larger than those previously characterized.

The cloning and molecular characterization of two of the de novo generated DI RNAs at 2 different stages of sequence evolution has been completed and the results have provided important clues about minimal cis-acting sequences important in DI multiplication. A summary of thes: key features are described and illustrated in Figure 5 of the grant.

OBJECTIVE 4) To perform in vitro mutagenesis experiments on biologically competent DI clones with the objective of identifying regions of DI RNA sequence important for replication, encapsidation and symptom attenuation.

The availability of an infectivity system for producing DI transcripts in vitro will provide us with the opportunity to begin the process of constructing and evaluating biologically active mutant transcripts to test specific functions. In addition, our sequence comparison studies have identified invariant and variant regions in the DI populations that can direct our mutagenesis efforts. These results coupled with the ability to evaluate the biological activity of transcripts derived from early stages in the evolution of de novo generated DIs has provided further clues 10 assist in the mutagenesis. The details are not provided here because some experiments are ongoing and will continue into the new grant period.

Assay for Virus Replication: In the past year, we have developed the capability to monitor the rate of replication of DI and helper virus in proloplasts (Jones et al., 1990). This was an important study because it clearly demonstrated that the primary effect of the DI

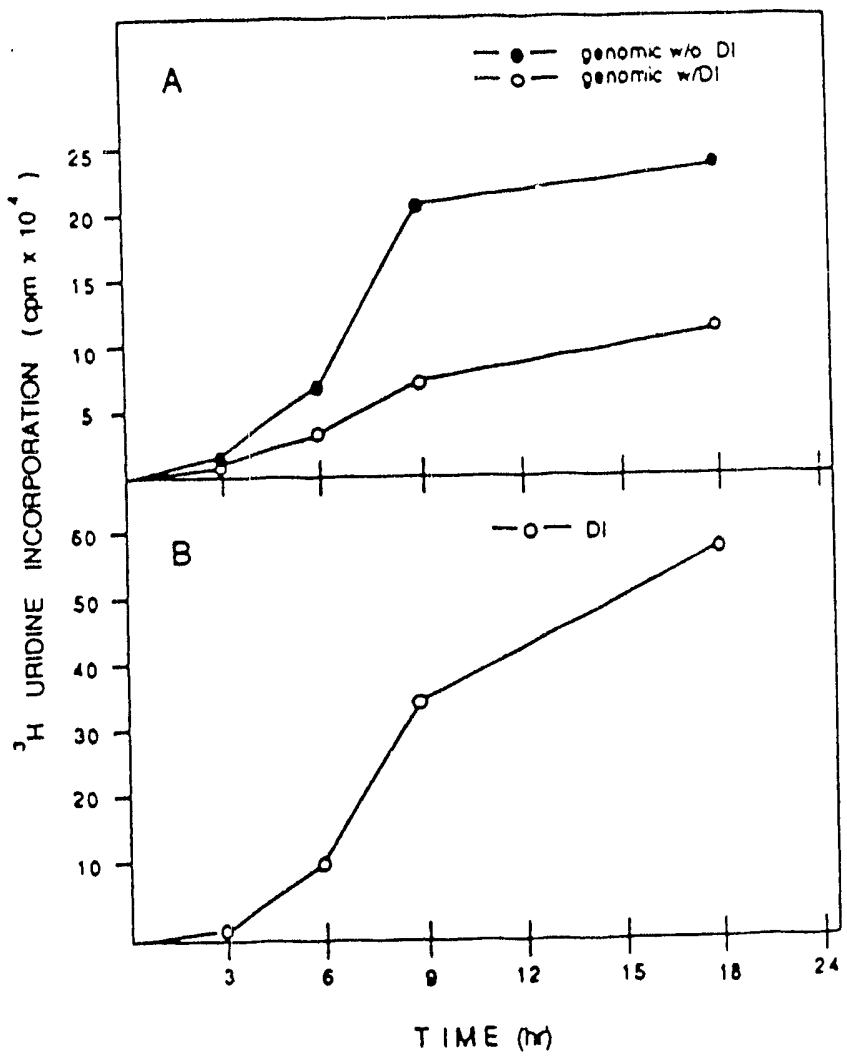

Figure 2. Quanlificalion of incorporation of $\mathrm{H}$ H.uridine inlo viral and DI RNAs accummulaling during infection of proloplasls 
genome during replication. The development of this protoplast assay system was an important step toward being able to study replication deficient mutants because we can direclly monitor the effect of mutation on replication competency. An example of the effect of the presence of DI replication on helper virus synthesis is illustrated in the northern blot shown as Fig. 1 of the grant proposal. The ability to quantitatively monitor rates of synthesis of viral specific RNAs and DIs as presented in Figure 2 provides an invaluable tool for addressing the effect of specific mutations on nelper virus synthesis in the proposed experiments.

Assay for Viral Encapsidation Sequences: Another important assay in addressing sequence function in mutant DIs is the ability to identify encapsidation signals using in vitro binding assays. We have developed such an assay for the TCV DI RNA described in Li et al., (1989). We hope to be able to extend this assay to the study of the TBSV DIs for which we now have infectious clones in an effort to identify sequences important in coat protein recognition and hence encapsidation.

OBJECTIVE 5) To transform plants with Ti plasmid constructs to evaluate the resistance of transgenic plants expressing DI related transcripts to TBSV infection.

Progress in this area has been slow because we have not been able to initiate the necessary effort with available personnel. We did, however, initiate some pilot experiments in the first 2 years of the proposal which involved the transformation of tomato with TBSV coat protein gene constructs. We did these experiments cooperatively with Dr. B.J. Staskawicz. Transgenic plants have been produced and evaluated by Southern blot analyses to identify lines harboring TBSV specific sequences. We are, however, more interested in evaluating resistance mediated by expressing DI RNAs in transgenic plants because these could provide a more effective and durable form of resistance than that mediated by coal protein. Such comparisons cannot be made, however, until we test both approaches. We have completed construction of Ti plasmid vectors harboring monomer constructs of DI sequences. We feeh, however, that initiation of the plant transformation experiments will require additional support in order to proceed at a good pace. Regeneration, characterization of DI expressing plants and greenhouse trials would require considerably more effort than can be provided by a single post-doc. As a consequence, we have obtained additional non-DOE support from the California Fresh Market Tomato Advisory Board for this aspect of the research.

\section{Papers Resulting from DOE Support since 1988 (" reprint/preprint attached)}

*1) Hearne, P., Knorr, D.A., Hillman, B.I., and Morris, T.J. 1990. The complete genome structure and infectious RNA synthesized from clones of tomato bushy stunt virus. Virology 177: (in press).

*2) Jones, R.W., Jackson, A.O. and Morris, T.J. 1990. Defective interfering RNAs and elevated temperatures inhibit replication of tomato bushy stunt virus in inoculated protoplasts. Virology 176: 539-545.

*3) Morris, T.J., and Knorr, D.A. 1990. Defective Interfering RNAs Associated with Plant Virus Infections. in Positive Strand RNA Viruses, Rueckert \& Brinton, ASM Publications (in press)

*4) Hillman, B.I., Hearne, P., Rochon, D'Ann., and Morris, T.J. 1989. Organization of the tomato bushy stunt virus genome: characterization of the coat protein gene and 3' terminus. Virology 169:4252.

5) Morris, T.J. and Hillman, B.I. 1989. Defective Interfering RNAs associated with a plant Tombusvirus. in Molecular Biology of Plant-Pathogen Interactions. UCLA symposia on molecular 
and cellular biology, New series, Volume 101, pp 185-197, Edilors B. Staskawicz, P. Ahlquist and O. Yoder. Alan R. Liss., New York, N.Y.

6) Li, X. H., Heaton, LA, Morris, T.J. and Simon A.E. 1989. Turnip Crinkle Virus Defective Interfering RNAs Intensify Viral Symploms and are Generated de novo. PNAS (USA) 86: 9173-9177.

7) Knorr, D.A and Morris, T.J. Origin and evolution of defective interfering RNAs of tomato bushy stunt virus. in Plant Molecular Biology, Ed. R. Herrmann; Plenum, (in press).

\section{Abstracts and Conference Proceedings since 1988}

1) Morris, T.J. and Hillman, B.I. 1988. Defective interfering RNAs of a small RNA plant virus. J. Cellular Biochem. Supplement 12C: 237.

2) Goldstein, A.L, and Morris, T.J. 1988. Characterization of a temperature induced RNA in tomato bushy stunt virus infections. Phytopathology 78: 1600.

3) Hearne, P.Q., Hillman, B.I., and Morris, T.J. 1988. Characterization of the coat protein gene of tomato bushy stunt virus. Phytopathology 78: 1600 .

4) Knorr, D.A., and Morris, T.J. 1988. Molecular characterization of defective interfering RNAs of tomato bushy stunt virus. Phytopathology 78: 1601.

5) Morris, T.J., Mullin, R., Hearne, P., and Hillman, B. 1988. De novo generation of tomato bushy stunt defective interfering RNAs. Phytopathology 78: 1610.

6) Morris, T.J. Defective interfering KNAs associated with plant virus infections. 2nd International Symposium on Positive Strand Viruses, Vienna, June 1989.

7) Knorr, D. A. and Morris, T.J. Molecular characterization of a population of defective interfering RNAs of tomato bushy stunt virus. Proceedings of the American Society of Virology, London, Canada, July 1989.

8) Knorr, D.A, \& Morris. T.J. 1989. Sequence analysis of defeclive interfering RNAs of tomato bushy stunt virus. Phytopathology 79: 1195.

9) Jones, R.W., Jackson, A.O. \& Morris, T.J. 1990. Inhibition of tomato bushy stunt virus by defective interfering particles in tobacco protoplasts. Phytopathology 79:1174.

\section{DISCLAIMER}

This report was prepared as an account of work sponsored by an agency of the United States This report was prepared as an account of work sponsored by a employees, makes any warranty, express ur implied, or assumes any legal liability or responsibility for the accuracy, completeness, or usefulness of any information, apparatus, product, or process disclosed, or represents that its use would not infringe privately owned rights. Referprocess disclosed, or represents that its use would not infringe private to any specific commercial product, process, or service by trade name, trademark. manufacturer, or otherwise does not necessarily constitute or imply its endorsement, recommendation, or favoring by the United States Government or any agency thereof. The views and opinions of authors expressed herein do not necessarily state or reflect those of the United States Government or any agency thereof. 
$\rightarrow$
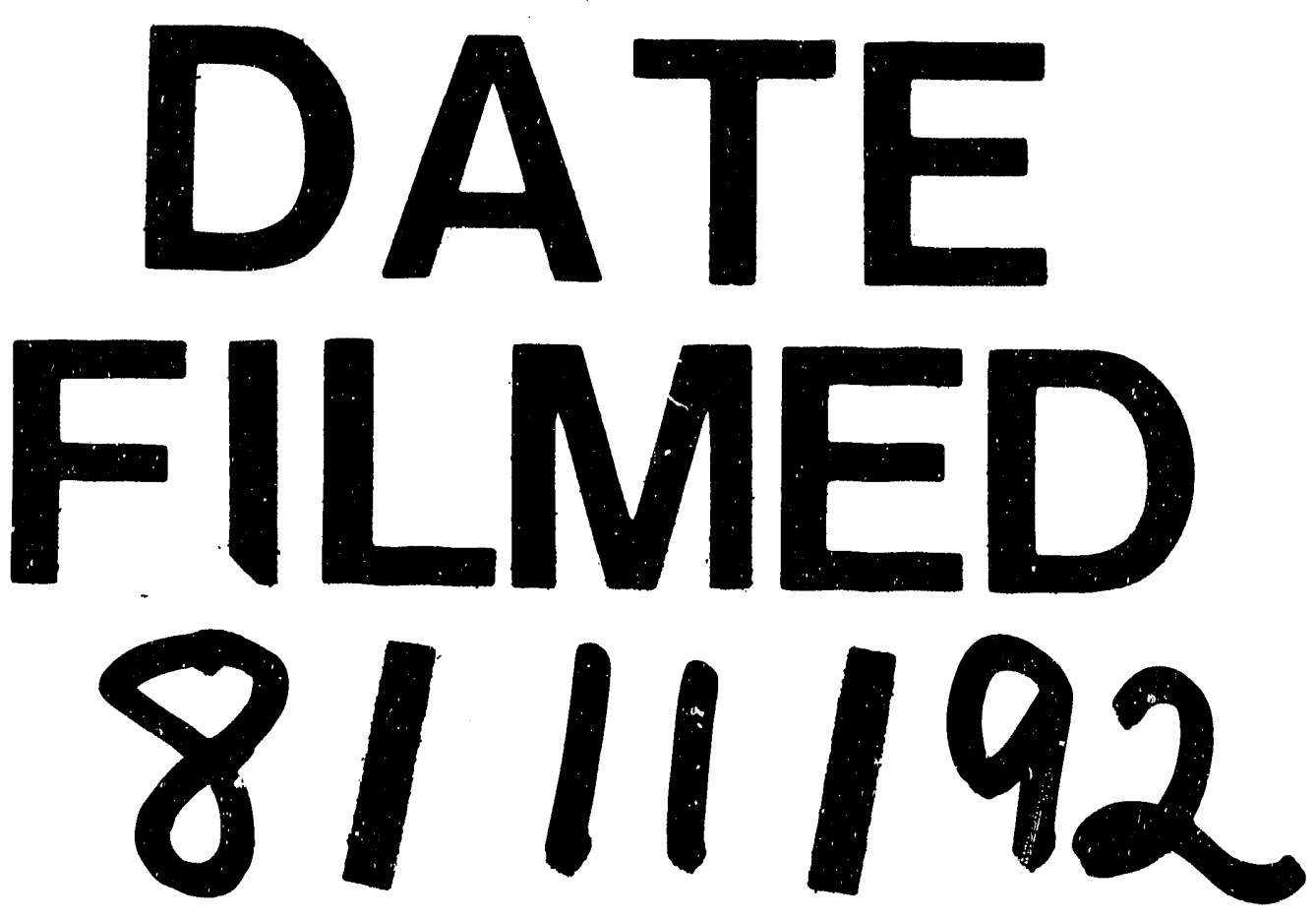
